\title{
Approximate controllability of multivalued stochastic fractional differential equations with Atangana-Baleanu-Caputo derivatives
}

\author{
Pallavi Bedi ${ }^{1}$, Anoop Kumar ${ }^{2}$, and Aziz Khan ${ }^{3}$ \\ ${ }^{1}$ Central University of Punjab \\ ${ }^{2}$ Central University of Punjab School of Basic and Applied Sciences \\ ${ }^{3}$ University of Peshawar
}

April 26, 2021

\begin{abstract}
This article aims to discuss the approximate controllability of multivalued impulsive stochastic fractional differential equations with ABC derivatives in Hilbert space. Firstly, with the help of stochastic analysis, theory of resolvent operators and the fixed point technique, we confirm the existence of mild solutions for the proposed control system. Secondarily, we show that the proposed system of equations is approximately controllable under a certain hypothesis. To confirm the applicability of the obtained results, an example is provided at the end of this paper.
\end{abstract}

\section{Hosted file}

Kumar Manuscript.pdf available at https://authorea.com/users/374349/articles/519535approximate-controllability-of-multivalued-stochastic-fractional-differential-equationswith-atangana-baleanu-caputo-derivatives 